\title{
Translational Behavioral Research in Respiratory Medicine
}

\author{
Bruce G. Bender, PhD; Mark S. Aloia, PhD; Allison E. Rankin, MPH, MSW; \\ and Frederick S. Wamboldt, MD
}

\begin{abstract}
Human behavior can prevent or invite disease and is a major determinant of treatment success. Consequently, many efforts have been directed toward developing interventions to promote behaviors essential to managing or preventing respiratory disease. The process of developing, testing, and disseminating health behavior interventions should closely follow the translational research paradigm. However, most behavioral investigators have failed to adequately apply the translational research paradigm to behavioral research. The final stage of translation, consisting of testing the effectiveness of interventions in broad clinical settings after efficacy has been demonstrated in randomized controlled trials, is too often omitted. Additionally, the important task of understanding why any given health behavior intervention succeeds with some people but fails to change behavior in others is inconsistently pursued and seldom used to develop the robust theories of behavior change needed to improve respiratory health. Bringing health behavior research through the full translational process has the potential to significantly enhance respiratory health with specific behavioral targets, including smoking cessation, obesity prevention, TB control, and adherence to a multitude of respiratory treatments.

CHEST 2011; 139(6):1279-1284

Abbreviations: $\mathrm{CER}=$ comparative effectiveness research; $\mathrm{PCT}=$ pragmatic clinical trial; $\mathrm{RCT}=$ randomized controlled trial
\end{abstract}

$\mathbf{H}$ uman behavior can prevent or invite disease and is a major determinant of the success of any planned treatment. Indeed, $40 \%$ of premature deaths in the United States are caused by human behaviors, including smoking, obesity, and alcohol use, with smoking accounting for the most premature deaths, killing 435,000 people in the United States each year. ${ }^{1}$ An additional 2.3 million Americans were living with smoking-induced respiratory or cardiovascular disease in 2006. ${ }^{2}$ Other behaviors that contribute to poorly

Manuscript received September 21, 2010; revision accepted January 5, 2011.

Affiliations: From the Department of Pediatrics (Dr Bender and Ms Rankin) and Department of Psychosocial Medicine (Drs Aloia and Wamboldt and Ms Rankin), National Jewish Health, Denver, CO; and Department of Psychiatry (Drs Bender, Aloia, and Wamboldt), University of Colorado School of Medicine, Denver, CO.

Correspondence to: Bruce G. Bender, PhD, The Center for Health Promotion, National Jewish Health, 1400 Jackson St, Denver, CO 80206; e-mail: benderb@njhealth.org

(C) 2011 American College of Chest Physicians. Reproduction of this article is prohibited without written permission from the American College of Chest Physicians (http://www.chestpubs.org/ site/misc/reprints.xhtml).

DOI: $10.1378 /$ chest.10-2443 controlled respiratory disease include an unwillingness to seek medical attention when symptoms are present, poor adherence to treatment, poor diet, and physical inactivity.

Debate over health-care reform has increased awareness of the importance of changing behavior to improve population health and reduce health-care costs. $^{3}$ The need for behavioral interventions that can improve health and illness self-management is now well recognized and has produced many research initiatives. Translational research aims to facilitate more effective dissemination and uptake of scientific advances so that evidence-based strategies for prevention and treatment reach patients in all care settings. ${ }^{4}$ Use of the translational research model to guide behavioral health research has been at best inconsistent. We believe that health behavior research can follow the translational research model and contribute significantly to accomplishing the national objectives of translational research (http://nihroadmap. nih.gov). This article discusses the five steps that can guide behavioral translational research to promote respiratory health (Fig 1). 


\section{Conduct Observational Studies of HeALTH BEHAVIOR}

The essential first step in behavioral health research is to document which behaviors are exhibited, by whom, when, and under what conditions. Using smoking behavior as an example, attempts to help people stop smoking began more than one-half a century ago. In the 1960s, the Centers for Disease Control and Prevention began surveying Americans about their health behavior, including documentation of cigarette smoking. In 1965, $42 \%$ of US adults reported themselves to be smokers. Concerns about the impact of smoking on health led to a multifaceted campaign of public education and awareness that began with the creation of a Surgeon General advisory committee appointed by President Kennedy. Smoking rates have slowly dropped since that time to $21 \%$ in the United States. ${ }^{5}$ Such research may include epidemiologic studies that record rates of behaviors across populations and observational studies that provide more specific information about the factors that predict behavior.

\section{Develop Models of Health Behavior}

Once observational studies have documented the frequencies and predictors of specified health behaviors, this information is used to build models, or theories, that explain the development of either detrimental or helpful health-related behaviors. Such models translate theory to the bedside by allowing behavioral scientists to develop and test theory-driven interventions that change people's health behavior. Health behavior research activities often are directed at testing and refining clinically useful strategies for behavior change by addressing the key factors from the model that determine health behavior. For example, if a model asserts that the patient's perception of imbalance between perceived treatment cost and benefit is a key factor underlying treatment nonadherence, then the intervention must address these perceptions. The model may be tested with an intervention that seeks to influence the patient's perception, and based on the outcomes, the investigators may gather evidence that leads to a refinement of the model and the intervention. One of the earliest, longest-standing, and most used theories of health behavior has been the Health Beliefs Model. ${ }^{6}$ The theory attempted to explain and predict specific health-related behaviors based on patterns of beliefs about the behavior and its relationship to health conditions. Based on testing of the Health Beliefs Model through many studies, the core thesis of the modelthe probability that a person will change his or her behavior depends on perception of health risk and the potential for benefit from an action-was refined to include other key elements. The Health Beliefs Model has evolved and encouraged related theories of health behavior change, including social-cognitive theory, ${ }^{7}$ theory of reasoned action, ${ }^{8}$ and theory of planned behavior ${ }^{9}$; all have been used to plan health behavior interventions.

\section{Test Laboratory-Based \\ InTERVENTIONS TO CHANGE BEHAVIOR in RANdomized Controlled Trials}

Interventions developed from behavior change models must first demonstrate efficacy in randomized controlled trials (RCTs). Results from RCT studies then can be used to refine the models and the interventions they foster. Behavioral RCTs for smoking began to appear $>40$ years ago, number over $6,000,{ }^{10}$ and have included attempts to reduce smoking with strategies that included use of reward contingencies, ${ }^{11}$ group therapy,${ }^{12}$ desensitization, ${ }^{13}$ partner support, ${ }^{14}$ physician-delivered advice, ${ }^{15}$ and telephone-based counseling. ${ }^{16}$ Despite a strong base of RCT validation, most smokers will not receive one of these empirically supported interventions.

Behavioral RCTs have advantages and disadvantages. In general, RCTs often sacrifice external validity for internal validity. Well-controlled and standardized studies of patients recruited and consented for participation in research allow for assertions of causality with high levels of confidence (high internal validity). At the same time, the artificial nature of laboratory studies casts doubt on the generalizability of the findings to broad clinical settings (low external validity). Many patients, particularly those with comorbidities and relatively common confounding problems such as depression, are excluded from these trials. Patients willing to participate in research studies, and who are paid to do so, may not respond in the same manner to an intervention as others who receive the intervention in the course of routine clinical care.

\section{IDENTIFy AND LeVERAge the Moderators of Response to BeHAVIORAL INTERVENTIONS}

No behavioral intervention is effective with all participants, and many result in sustained change in only a minority. Among the 17 million adults who attempted to stop smoking in 1991, only about $7 \%$ were still abstinent 1 year later. ${ }^{17}$ The most recent guidelines for treating tobacco use and dependence, published by the Agency for Healthcare Research and Quality, reported a meta-analysis that included $>6,000$ published RCTs of smoking cessation interventions. ${ }^{10}$ 


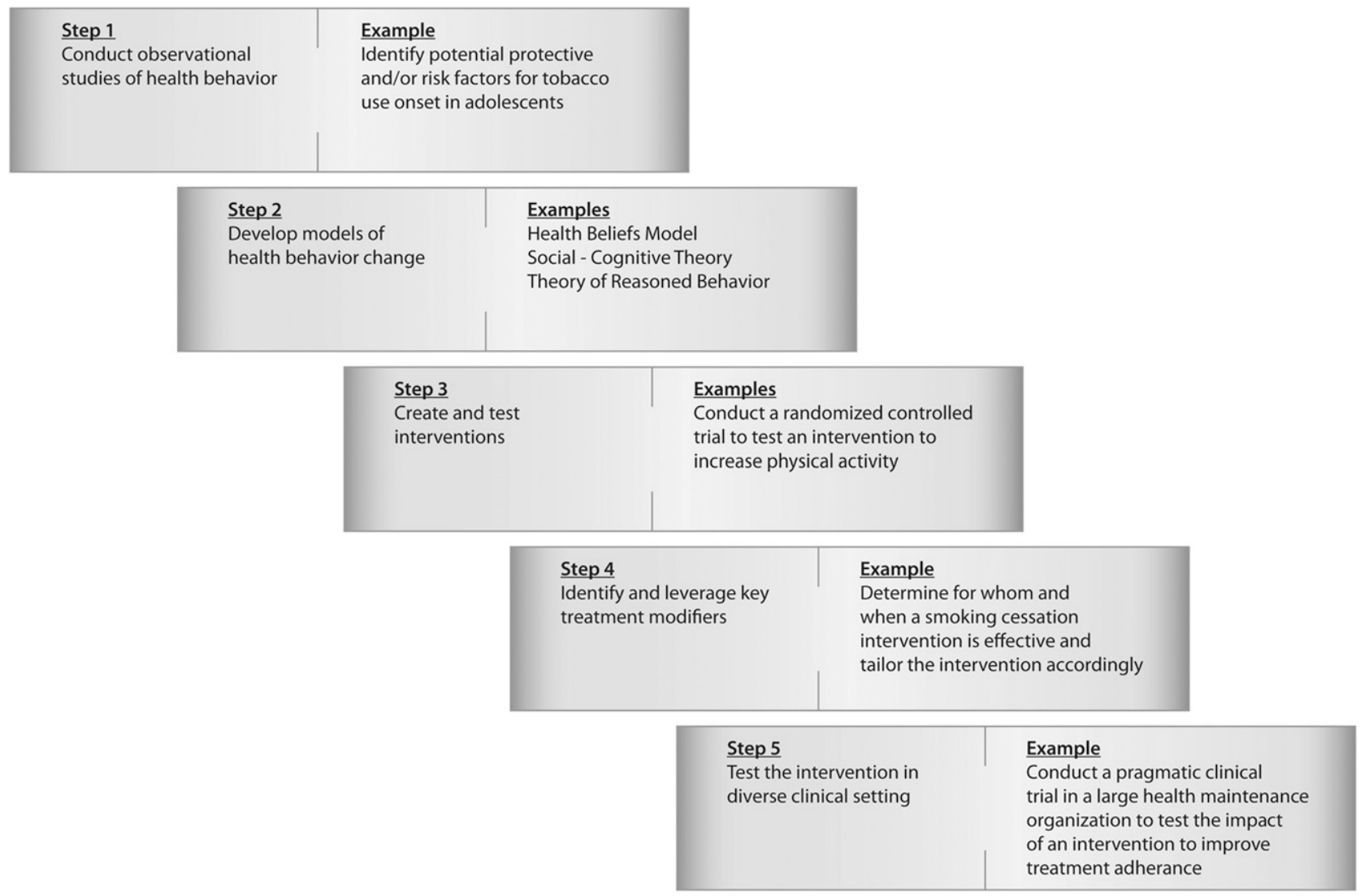

FIgURE 1. Translational sequence for health behavior research.

Composite evidence indicated that ORs of abstinence rates of at least 5 months were 1.2 for selfhelp, telephone counseling, and group counseling and 1.3 for individual counseling. ${ }^{10}$ Thus, approximately $30 \%$ of people who received an individually delivered behavioral intervention successfully quit smoking.

The expectation that any intervention will change behavior in all individuals is unrealistic, but understanding the moderating variables that influence treatment effects can assist in modifying the intervention to make it more effective for more people or to identify the targets for any given intervention. Moderating variables help to define the specific conditions under which the interventions demonstrate their greatest or poorest efficacy. ${ }^{18}$ Although broad interventions often target "low hanging fruit," that is, the subset people who are ready to change and respond positively, the potential for greater penetration and impact is lost without further tailoring. To achieve this tailoring, ongoing work must examine treatment moderators. ${ }^{18}$ Hence, a smoking cessation intervention designed specifically for adolescents may be more effective for an adolescent smoker than one designed for older adults. Investigators often choose to control for these baseline factors to increase internal validity. ${ }^{19}$ Alternatively, such variables may be used to tailor the intervention to various subgroups. For example, a smoking cessation intervention may be more effective if it includes attempts to encourage social support of the smoker attempting to quit. Recognizing that smokers with insufficient support from friends and family are less successful, one RCT intervention demonstrated that pregnant smokers who received an intervention that included the participation of a friend were significantly more likely to quit than those who received the intervention without friend participation. ${ }^{20}$

Intervention moderators should not be confused with predictors of behavior. Many adherence studies have examined variables that correlate with adherence, but few have assessed the variables that predict change in adherence in response to an intervention. It is known, for example, that patients who are adolescents, ${ }^{21}$ elderly, ${ }^{22}$ low income, ${ }^{23}$ distrustful of medical professionals, or psychologically distressed ${ }^{24}$ or who have low health literacy ${ }^{25}$ or disease knowledge ${ }^{26}$ are less likely to adhere to a long-term medication regimen. These correlations can provide hypotheses about which variables to study as potential treatment 
effect moderators. However, they do not invariably predict intervention response or direct which type of intervention is best matched with subgroups of patients. Assessment of the moderators of adherence can be accomplished in RCTs and may include participant demographic characteristics as well as other psychologic variables, such as personal beliefs and attitudes toward risk. Results from such investigations have revealed, for example, that particular smoking cessation interventions have differential impacts on tobacco abstinence based on age, ${ }^{27}$ education level, ${ }^{28}$ gender, ${ }^{29}$ acculturation, ${ }^{30}$ or psychologic distress. ${ }^{31}$

The identification of moderators that affect the success of the intervention and, consequently, can be used to guide further tailoring of the intervention is at best infrequent in behavioral RCTs. Although using these treatment modifiers to improve treatment effectiveness is an important step forward, further tailoring alone does not ensure that the intervention will translate well into diverse clinical settings. Both steps-treatment tailoring and implementation in diverse practice settings - are necessary if significant health behavior change in large numbers of people is to be accomplished.

\section{Assess the Intervention in Diverse Clinical Practice Settings}

An intervention that appears effective in an RCT may not translate into effectiveness under real-world conditions. Health behavior researchers too often accept a diffusion model, which relies on absorption and implementation of intervention techniques by health behavior specialists with the knowledge and time to successfully move the strategy through the last translational transition from clinical trials to practice implementation. ${ }^{32}$ In fact, most behavior change interventions that are tested in RCTs will never be adopted into health-care settings. Four barriers underlie this failure. First, although some healthcare settings may include health behavior specialists, most do not, leaving the task of motivating patients to change their behavior to busy physicians, nurses, and physician assistants. Second, many behavioral interventions are time, labor, and cost intensive and therefore are unfeasible within most health-care systems. Third, RCTs often are only modestly effective, and moderators/mediators rarely are identified, leaving clinicians and researchers feeling less confident in their applicability to the clinical setting. Fourth, RCTs typically exclude patients who have comorbid medical or psychologic disorders or who do not demonstrate high levels of adherence during a run-in period, thereby failing to test the intervention on a complex combination of patients who comprise the patient population in many clinical settings.
Although testing behavioral health interventions in clinical practice requires moving beyond traditional RCTs, it is still possible to use a controlled, randomized study design in what is now labeled a practical or pragmatic clinical trial (PCT). ${ }^{33}$ The identifying characteristics of PCTs are (1) comparison of clinically relevant interventions, (2) inclusion of a variety of participants recruited from diverse practice settings, and (3) use of a broad range of health outcomes, including patient quality of life. PCTs often test interventions within a population of patients who receive the intervention in their own clinical care setting. Thus, the traditional RCT approach of recruiting patients, excluding those who do not qualify, and engaging in study procedures that do not reflect routine care is altered. Further, the consent process is adapted such that the consent often is obtained in an abbreviated manner that does not require the participant to make an extra visit to meet in a formal interaction that suggests that what is about to happen is special and differs from their usual clinical care.

As an example, one PCT tested a smoking-cessation intervention in a large health maintenance organization. The subject pool included 2,234 smokers who were about to undergo gastrointestinal outpatient surgery, as identified from the electronic medical record system, and were sent a letter describing the study from the chief of preventive medicine. The letter was accompanied by an opt-out postcard that respondents could return to decline participation. Those who did not return the postcard received a telephone call wherein the researchers described the study procedures and obtained verbal consent. Eligible participants were subsequently randomized to an intervention or usual care program. The intervention group received four counseling telephone calls and five newsletters, including one tailored to specific patient groups on the basis of gender, smoking rate, and responses to a self-efficacy questionnaire. ${ }^{34}$ The study was a theory-anchored intervention that was based on data from previous RCTs and studies of key mediators, included a diverse patient population in a large clinical setting, and constituted a system intervention that did not require additional time from clinic healthcare providers.

Additionally, recent emphasis on comparative effectiveness research (CER) has emerged from the recognition that evidence of the relative benefits and harms of available treatments often is inadequate to fully inform health-care decisions. ${ }^{35}$ Further, the relative effectiveness of treatments may vary among subgroups of patients, many of whom were not included in traditional efficacy trials. CER studies frequently are designed to include end points important to patients, such as quality of life. CER and PCT studies introduce more variability, or "noise," into the equation 
and therefore typically require larger sample sizes. Institutional review boards often are required to consider more flexible approaches to consent when large groups of patients may be randomized under circumstances where study risk is estimated to be very low and consent is obtained by sending a letter to potential participants who are given the opportunity to opt out of the program. The potential benefits of such research are a better understanding of the heterogeneity of treatment effects and how best to deliver care in real-world settings. ${ }^{36}$ Recognizing the importance of this potential, the American Recovery Reinvestment Act of 2009 provided $\$ 1.1$ billion in funding to support CER, and in 2010, the Patient Protection and Affordable Care Act included funding guidance for increased CER research.

Other important initiatives have addressed time constraints that limit the capacity of health-care providers to deliver health behavior change interventions. These efforts include the growth of practice-based research networks in primary care ${ }^{37}$ and the ongoing expansion of continuous quality improvement in health-care settings. ${ }^{38}$

\section{ConClusions}

The successful application of the translational model to health behavior research offers a promise that has not yet been delivered. Key behaviors targeted for change include increased physical activity and treatment adherence, improved dietary intake, and smoking cessation. Despite the emergence of some interventions that can change these behaviors, health behavior research has not succeeded in creating interventions that are cost-effective and easily translated into diverse health-care settings or that can be delivered by a variety of health-care professionals. To achieve a much broader impact, two key approaches must be adopted. Identification of health behavior intervention moderators will shed light on how to refine and tailor theories and interventions to affect the largest possible number of patients. Second, the capacity of the intervention to be broadly and costeffectively adopted in diverse health-care settings must be established. With these steps, health behavior research can proceed through the full translational research process and deliver its potential to significantly enhance respiratory health.

\section{ACKNOWLEDGMENTS}

Financial/nonfinancial disclosures: The authors have reported to CHEST the following conflicts of interest: Dr Aloia receives grant support from the National Institutes of Health, is a paid employee for Philips Respironics, and holds stock in the parent company, Philips Healthcare. Dr Wamboldt has received funding from pharmaceutical firms and grant support from governmental and private foundations. Dr Bender and Ms Rankin have reported that no potential conflicts of interest exist with any companies/ organizations whose products or services may be discussed in this article.

\section{REFERENCES}

1. Schroeder S. Shattuck lecture. We can do better-improving the health of the American people. N Engl J Med. 2007; 357(12):1221-1228

2. Mannino DM, Homa DM, Akinbami LJ, Ford ES, Redd SC. Chronic obstructive pulmonary disease surveillance-United States, 1971-2000. MMWR Surveill Summ. 2002;51(16):1-16.

3. Brook RH. Rights and responsibilities in health care: striking a balance. JAMA. 2010;303(22):2289-2290.

4. Dougherty D, Conway PH. The "3T's" road map to transform US health care: the "how" of high-quality care. JAMA. 2008;299(19):2319-2321

5. Centers for Disease Control and Prevention; National Center for Health Statistics. Faststats: smoking. 2010. http://www. cdc.gov/nchs/fastats/smoking.htm. Accessed January 29, 2011.

6. Rosenstock IM. Why people use health services. Milbank Mem Fund Q. 1966;44(3):94-127.

7. Bandura A. Social cognitive theory. In: Vasta R, ed. Annals of Child Development. Six Theories of Child Development. Vol 6. Greenwich, CT: JAI Press; 1989:1-60.

8. Sutton S, McVey D, Glanz A. A comparative test of the theory of reasoned action and the theory of planned behavior in the prediction of condom use intentions in a national sample of English young people. Health Psychol. 1999;18(1): $72-81$.

9. Ajzen M. Perceived behavioral control, self-efficacy, locus of control, and the theory of planned behavior. J Appl Soc Psychol. 2002;32(4):665-683.

10. Fiore M, Jaén C, Baker T, et al. Treating Tobacco Use and Dependence: 2008 Update [Updated Clinical Practice Guideline]. Rockville, MD: US Department of Health and Human Services; 2008.

11. Zagona SV, Zurcher LA Jr. The relationship of verbal ability and other cognitive variables to the open-closed cognitive dimension. J Psychol. 1965;60:213-219.

12. Guilford JS. Group treatment versus individual initiative in the cessation of smoking. J Appl Psychol. 1972;56(2):162-167.

13. Lowe MR, Green L, Kurtz SM, Ashenberg ZS, Fisher EB Jr. Self-initiated, cue extinction, and covert sensitization procedures in smoking cessation. J Behav Med. 1980;3(4):357-372.

14. Coppotelli HC, Orleans CT. Partner support and other determinants of smoking cessation maintenance among women. J Consult Clin Psychol. 1985;53(4):455-460.

15. Ockene J, Hosmer D, Williams J, et al. The relationship of patient characteristics to physician delivery of advice to stop smoking. J Gen Intern Med. 1987;2(5):337-340.

16. Orleans C, Schoenbach V, Wagner E, et al. Self-help quit smoking interventions: effects of self-help materials, social support instructions, and telephone counseling. $J$ Consult Clin Psychol. 1991;59(3):439-448.

17. Centers for Disease Control and Prevention (CDC). Smoking cessation during previous year among adults-United States, 1990 and 1991. MMWR Morb Mortal Wkly Rep. 1993;42(26):504-507.

18. Kraemer HC, Wilson GT, Fairburn CG, Agras WS. Mediators and moderators of treatment effects in randomized clinical trials. Arch Gen Psychiatry. 2002;59(10):877-883.

19. Kraemer HC, Frank E, Kupfer DJ. Moderators of treatment outcomes: clinical, research, and policy importance. JAMA. 2006;296(10):1286-1289. 
20. Higgins ST, Heil SH, Badger GJ, Skelly JM, Solomon LJ, Bernstein IM. Educational disadvantage and cigarette smoking during pregnancy. Drug Alcohol Depend. 2009;104(suppl 1): S100-S105.

21. Bender BG. Overcoming barriers to nonadherence in asthma treatment. J Allergy Clin Immunol. 2002;109(6 suppl): S554-S559.

22. Apter AJ, Boston RC, George M, et al. Modifiable barriers to adherence to inhaled steroids among adults with asthma: it's not just black and white. J Allergy Clin Immunol. 2003;111(6):1219-1226.

23. DiMatteo MR, Lepper HS, Croghan TW. Depression is a risk factor for noncompliance with medical treatment: metaanalysis of the effects of anxiety and depression on patient adherence. Arch Intern Med. 2000;160(14):2101-2107.

24. Bender B, Milgrom H, Rand C, Ackerson L. Psychological factors associated with medication nonadherence in asthmatic children. J Asthma. 1998;35(4):347-353.

25. Osman L, Russell I, Friend J, Legge J, Douglas J. Predicting patient attitudes to asthma medication. Thorax. 1993;48(8): 827-830.

26. Ho J, Bender B, Gavin L, O’Conner S, Wamboldt M, Wamboldt F. The relationships between asthma knowledge, treatment adherence, and outcome. J Allergy Clin Immunol. 2003;111(3):498-50.

27. Murray RP, Connett JE, Rand CS, Pan W, Anthonisen NR. Persistence of the effect of the Lung Health Study (LHS) smoking intervention over eleven years. Prev Med. 2002; 35(4):314-319.

28. Strecher V, McClure J, Alexander G, Chakraborty B, Nair V, Konkel J. The role of engagement in a tailored web-based smoking cessation program: randomized controlled trial. J Med Internet Res. 2008;10(5):e36.
29. Monsó E, Campbell J, Tonnesen P, Gustavsson G, Morera J. Sociodemographic predictors of success in smoking intervention. Tob Control. 2001;10(2):165-169.

30. Webb M. The moderating role of acculturation in culturally specific interventions. Psychol Addic Behav. 2008;22(4): 592-596.

31. Carmody T, Duncan C, Simon J, Solkowitz S, Huggins J, Lee S, Delucchi K. Hypnosis for smoking cessation: a randomized trial. Nicotine Tob Res. 2008;10(5):811-818.

32. Estabrooks PA, Glasgow RE. Translating effective clinic-based physical activity interventions into practice. Am J Prev Med. 2006;31(4suppl):S45-S56.

33. Tunis S, Stryer D, Clancy C. Practical clinical trials: increasing the value of clinical research for decision making in clinical and health policy. JAMA. 2004;290(12):1624-1632.

34. Glasgow RE, Estabrooks PA, Marcus AC, et al. Evaluating initial reach and robustness of a practical randomized trial of smoking reduction. Health Psychol. 2008;27(6):780-788.

35. Luce BR, Kramer JM, Goodman SN, et al. Rethinking randomized clinical trials for comparative effectiveness research: the need for transformational change. Ann Intern Med. 2009, 151(3):206-209.

36. Krishnan J, Schatz M, Apter A. A call for action: comparative effectiveness research in asthma. J Allergy Clin Immunol. 2010;127(1):123-127.

37. Nutting P, Baier M, Werner J, Cutter G, Reed F, Orzano A. Practice patterns of family physicians in practice-based research networks: a report from ASPN. Ambulatory Sentinel Practice Network. J Am Board Fam Pract. 1999;12:278-284.

38. Spencer E, Swanson T, Hueston WJ, Edberg DL. Tools to improve documentation of smoking status. Continuous quality improvement and electronic medical records. Arch Fam Med. 1999;8(1):18-22. 